\title{
Is Mathematical Logic Really Necessary in Teaching Mathematical Proofs?
}

\author{
By Michael Aristidou*
}

\begin{abstract}
As it is already observed by mathematicians and educators, there is a discrepancy between the formal techniques of mathematical logic and the informal techniques of mathematics in regards to proof. We examine some of the reasons behind this discrepancy and to what degree it affects doing, teaching and learning mathematics in college. We also present some college students' opinions about proofs, and we briefly observe the situation in Greek and GreekCypriot high schools in which mathematical logic is part of the curriculum. Finally, we argue that even though mathematical logic is central in mathematics, its formal methods are not really necessary in doing and teaching mathematical proofs and the role of those formalities has been, in general, overestimated by some educators.
\end{abstract}

Keywords: formal, logic, proof, student, teacher.

\section{Introduction}

In several colleges, some parts of mathematical logic (i.e. sets, propositional logic, and predicate logic) are usually taught in the early chapters of a discrete mathematics class, in order to prepare the students for the important chapter on proofs and proving techniques. Yet, most likely, students have already been exposed to proofs before the above-mentioned course in other mathematics courses or even in high school. Mathematical logic is to sharpen the logical and analytical skills of a student as these are necessary for the understanding and learning of mathematical proofs. Mathematical logic though is characterized by its symbolic presentation and formal rules. Mathematics, on the other hand, combines mathematical symbolism and natural language and its methods are rigorous yet less formal.

Historically, logic is associated with Aristotle and his work the Organon in which he introduced terms like "propositions" and "syllogisms", the basics on categorical and hypothetical syllogism, and modal and inductive logic. It is also associated with the Stoics and their propositional logic, and their work on implication. Syllogistic logic and propositional logic led later to the development of predicate logic (or first order logic, i.e. the foundational logic for mathematics) by Frege and Hilbert in the $19^{\text {th }}$ century. As Ferreiros said:

"First-order logic emerged as an analysis of the most fundamental basis for the notion of mathematical proof. To put it otherwise, it emerged as the logic that is necessary

*Instructional Assistant Professor, Texas A\&M University at Galveston, USA. 
and sufficient for codifying mathematical proofs, axiomatizing mathematical theories, and studying their metatheory." (Ferreiros, 2001, p. 479)

Predicate logic is also the foundation of modern mathematical logic. The latter is a subfield of mathematics that includes fields such as set theory, model theory and proof theory, and its primary interests are the foundations of mathematics and theoretical computer science.

The interest in the foundations began in the $19^{\text {th }}$ century with the development of axiomatic frameworks for geometry and arithmetic by Hilbert and Peano respectively. That led in the early $20^{\text {th }}$ century to three main philosophies regarding the foundations of mathematics, namely Logicism, Formalism and Intuitionism, none of which adequately accounts for those foundations. Gödel pointed out the issues of consistency and completeness related to provability in general formal systems. Nevertheless, most mathematics can be formalized in terms of sets, and set theory serves nowadays as its foundation. In real mathematical practice though rarely one adheres to set-theoretical foundations to validate or refute mathematical questions. Each mathematical field has its own tools and methods and with general logical framework the predicate logic explores its own questions, proves its own theorems, and establishes connections between fields. Even though mathematical logic is central in mathematical practice, its strict symbolism and formal rules are rarely used in mathematics, whose mathematical symbolism, language and methods are rigorous yet less formal.

In the subsequent sections, we will look at the differences between the formal techniques of mathematical logic and the informal techniques of mathematics in regards to proof. We will examine how it affects doing, teaching and learning mathematics, give some examples, and present some college students' opinions about proofs. We will also see what/when logic is taught in Greek and GreekCypriot high schools as it is part of the school curriculum.

\section{What is Mathematical Proof?}

Even though there is no complete agreement among mathematicians on what constitutes a mathematical proof, it is accepted by most that proof is a central activity in mathematics. A proof is basically a line of reasoning that mathematicians would employ in order to convince someone about the truth of a mathematical statement. A mathematical proof is usually written in an algerbraic-symbolic form, mixed with natural language, and it has among others the following basic objectives: (a) verification, (b) discovery, (c) explanation, (d) communication, (e) challenge, (f) systematization. This is what is usually characterized as "informal proof" and what most practicing mathematicians usually do and understand as proof. As Hersh says:

"Practical mathematical proof is what we do to make each other believe our theorems." (Hersh, 1997, p. 49) 
A proof also could be re-phrased, proved differently, refined, completed, etc. All the above play a crucial role in the mathematical progress.

Students often learn about the different types of proof techniques, such as direct proof, proof by cases, proof by contradiction, etc., which are based on some basic logical rules of inference such as modus ponens, modus tollens, resolution, etc., and their extensions in predicate logic.

Example: If $n$ is an odd integer, then $n^{2}$ is odd.

Proof: Let $n$ be an odd integer. Then, there exists an integer $k$ such that $n=2 k+1$. Squaring both sides of the equation, we have that:

$n^{2}=(2 k+1)^{2}=4 k^{2}+4 k+1=2\left(2 k^{2}+2 k\right)+1=2 \lambda+1$, where $\lambda=2 k^{2}+2 k$.

Hence, by the definition of odd, we have that $n^{2}$ is odd.

The above proof is a typical (informal) mathematical proof, and is based on the modus ponens. That is, on the logical schema:

$$
\begin{aligned}
& \forall x\left[O(x) \rightarrow O\left(x^{2}\right)\right] \\
& O(n) \\
& \ldots \ldots \ldots \ldots \\
& \therefore O\left(n^{2}\right)
\end{aligned}
$$

where $O(x)=$ " $x$ is odd" and $x \in \mathrm{Z}$.

What about "formal proofs"? A formal proof (derivation) is a sequence of steps where from a given set of sentences (premises) one derives another sentence (conclusion) using the logical rules of inference. A formal proof has more of a syntactic nature, than semantic and employs deductive reasoning rather than other forms of reasoning. It is highly rigorous, recalls all relevant axioms and definitions, uses and manipulates logical symbols, and emphasizes the verification aspect of a proof, and not so the explanatory aspect. So, the previous example would be written formally as follows:

Example: If $n$ is an odd integer, then $n^{2}$ is odd.

Proof: 1. $O(x) \quad$ premise

2. $\exists z(x=2 z+1) 1$, definition of odd

3. $x=2 m+12$, existential instantiation

4. $x^{2}=2\left(2 k^{2}+2 k\right)+1 \quad 3$, algebra

5. $x^{2}=2 z+1 \quad 4$, existential generalization

6. $O\left(x^{2}\right) \quad 5$, definition of odd

7. $O(x) \rightarrow O\left(x^{2}\right) \quad$ 1-6, modus ponens

8. $\forall x\left[O(x) \rightarrow O\left(x^{2}\right)\right] \quad 7$, universal generalization 
Notice that some mathematicians claim that a proper proof is actually the formal proof, or at least that an informal proof is acceptable if a formal proof could in principle be constructed. As Rota says:

"A proof of a mathematical theorem is a sequence of steps which leads to the desired conclusion. The rules to be followed by such sequence of steps were made explicit when logic was formalized early in this century, and they have not changed since." (Rota, 1997, p. 183)

Finally, formal proofs are usually checked and constructed using computers and they are quite long (see Figure 1) and time consuming. For example, the proof of Kepler's Conjecture by Hales in 2006was more than 250 pages long (Hales \& Ferguson, 2006), and it took a group of 22 people more than 10 years to formalize the proof (Hales et al., 2017).

Figure 1. The proof of the irrationality of $\sqrt{2}$ in proof assistant Isabelle Main theorem

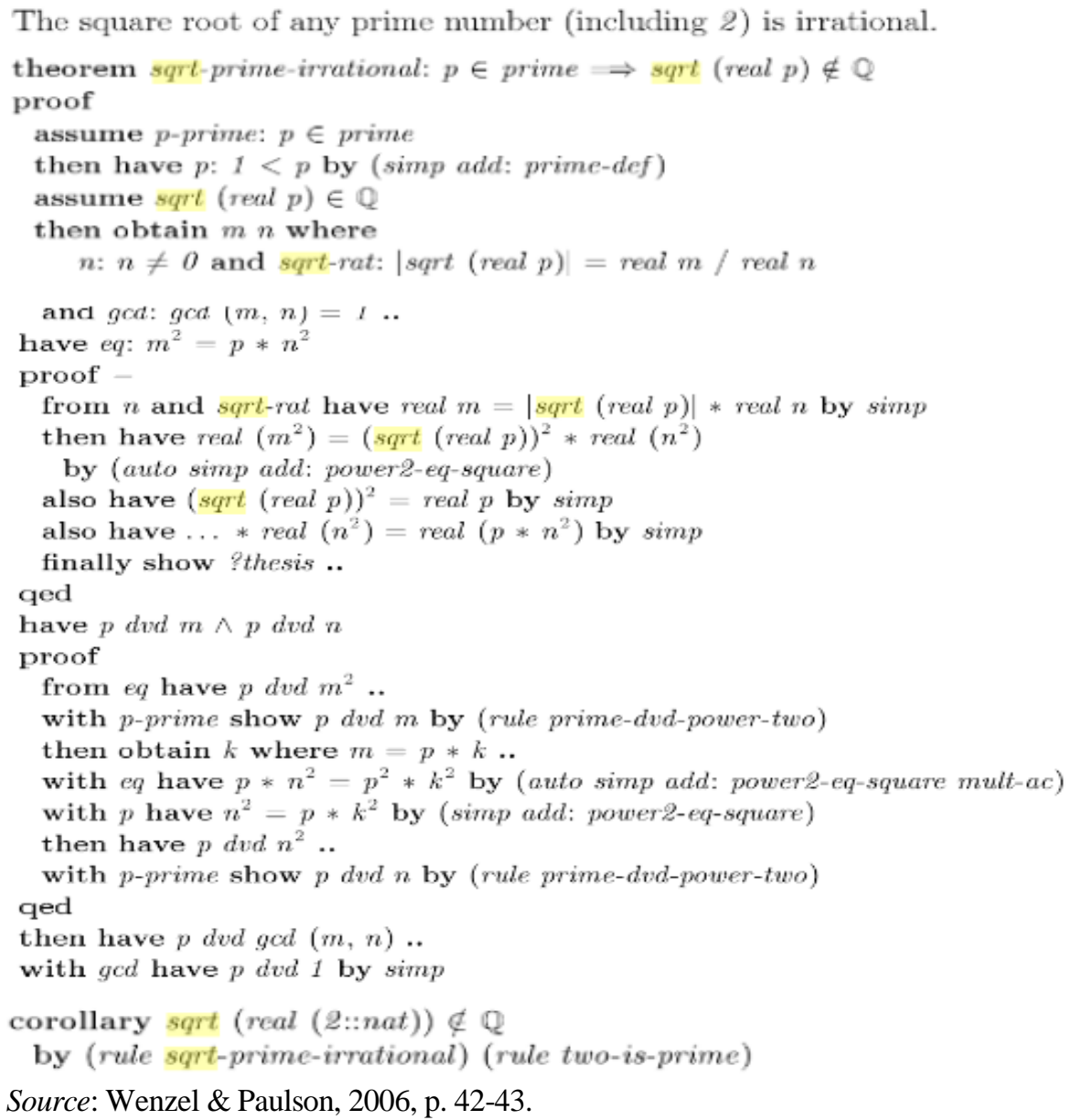

Source: Wenzel \& Paulson, 2006, p. 42-43. 


\section{Issues with Formal Logic}

Comparing the two proofs in the example above, one can see some quantitative and qualitative differences. First, the second proof is a bit longer and it can get much longer when the theorems get more interesting. Then, one notices that the second proof is not very explanatory or communicative. It is intended to deductively verify the theorem, and it reminds of a computer program. As a matter of fact, if the above proof were computer-performed, it would also get even longer as one would be required to input also all necessary definitions, axioms and calculations, in order to arrive to the conclusion. Finally, the second proof is not the way that mathematicians do and publish proofs in their field, neither is the way they teach their students in mathematics classes.

But why is that? There are several reasons. We outline some below.

\section{Epistemic Reasons}

On the practical level, making proofs unnecessarily longer, less readable, and harder to communicate, does not benefit the students or the teachers in terms of knowledge. Since proofs are central to the development and transfer of mathematical knowledge, they should be in a format that most understand, so students or teachers can communicate it to others and motivate discussions that could lead to further discoveries. On the theoretical level, could all mathematical statements be formalized and proved? Godel's Incompleteness Theorems impose some serious restrictions on provability within a formal system that is large enough to handle basic mathematics. Marfori argues quite convincingly that formal understanding of proof "yields an implausible account of mathematical knowledge, and falls short of explaining the success of mathematical practice" (Marfori, 2010, p. 261). She raises two important objections: one referring to the circularity of the notion of rigorous proof and one doubting formalism's explanatory power with respect to ordinary mathematical practice.

\section{Not Just Deduction}

Even though deductive inference is central in proofs and in mathematics in general, it is not the only type of inference in mathematical practice. Peirce considers three kinds of logical inference, namely deductive, inductive and abductive, which he sees as important stages in mathematical inquiry (Bellucci \& Pietarinen, 2018). Certainly, deduction allows one to move from some hypotheses to a conclusion, but hypotheses and conjectures must be formed in the first place. That can be done by induction and abduction by looking at some specific examples first, draw analogies, and then generalizing. Deduction, in mathematical inquiry, usually comes at the last stage as a way to verify certain observations. Polya $(1954 ; 1973)$ and Lakatos (1976) explain the process of mathematical discovery very clearly. For example, Polya lays down some steps for general problem solving that include: understanding the problem, experimenting, 
conjecturing, generalizing, trying to prove and proving or disproving. The steps before the proving step are what one would call the inductive/abductive stage ${ }^{1}$.

\section{Intuition also Necessary}

Clearly, logic is necessary for doing mathematics. But is it sufficient? As Hadamard said:

"[...] strictly speaking, there is hardly any completely logical discovery. Some intervention of intuition issuing from the unconscious is necessary at least to initiate the logical work." (Hadamard, 1954, p. 112)

In a completed proof, formal or informal, one rarely sees all the mathematical activity that preceded the proof. That activity might have included scattered thoughts, incomplete notes, calculations, drawing diagrams, experimenting, moments of inspiration, several failures, frustration, etc. All these activities are sometimes part of the mathematical process, yet they are not part of the logical process. And they are not characterized by the deductive nature that usually characterizes a proof. A proof seems to comprise all the above in an end result argument, and comes after the discovery. And, in general, logic seems to merely follow intuition.

\section{Not all are Computer Scientists}

In a computer science class, logic is covered not only to serve as a problem solving tool, but also, as Hein says:

"[...] for its use in formal specification of programs, formal verification of programs, and for its growing use in many areas such as databases, artificial intelligence, robotics, automatic reasoning systems, and logic programming languages." (Hein, 2010, p. vi)

Formal proofs are also covered, usually after informal proofs have been covered. As important as Hein's topics may be, they are not the primary interests in a mathematics course, even in a discrete mathematics course which is prerequisite to computer science. In mathematics course the emphasis falls on informal proofs, their structure and the information they convey, the relation of the proved theorems to other theorems, examples, historicals, and, of course, some applications to other sciences.

\footnotetext{
${ }^{1}$ Polya also explains the difference between induction and mathematical induction (a deductive process) and gives a nice example applying all the previously mentioned steps (1973, p.114-121). In particularly, he proves the theorem "The Sum of the First $n$ Cubes is a Square", showing all the previous steps and activity that led one to the theorem, doing calculations, using visuals, forming conjectures, etc.
} 


\section{Some Objections}

With the advancement of computers, programming and computer algebra systems in particular, some argue for the use of formal techniques in mathematics for philosophical but also pragmatic reasons. For instance:

"-To establish or refute a thesis about the nature of mathematics or related questions in philosophy.

-To improve the actual precision, explicitness, and reliability of mathematics." (Harrison, 2008, p. 1395)

Regarding the first point, Harrison justifies the formalization of mathematical proofs by appealing to the foundations of Mathematics. As he says:

"[...] the defining characteristic of mathematics is that it is a deductive discipline. Reasoning proceeds from axioms (or postulates), which are either accepted as evidently true or merely adopted as hypotheses, and reaches conclusions via chains of incontrovertible logical deductions." (Harrison, 2008, p. 1395)

As Harrison continues, in the past, informal methods caused ambiguities and errors $^{2}$, and informal proofs bearing the burden of being explanatory lost rigor and precision. Hence, it is only natural to utilize the deductive nature of mathematics and strive for formalizing proofs and presenting them in a "high-level" conceptual way. This way, there are no issues of uncertainty or errors and one is sure of what has been proved from given assumptions. A computer program could take over this process, as it has already done for several theorems, and help tremendously and change the mathematical practice.

The only problem though is that Harrison puts mathematics on narrow foundations. Mathematics is more than just deduction of statements and proof is just one of the stages in the mathematical activity ${ }^{3}$, as Lakatos (1976) and Polya (1954; 1973) nicely documented in their classic works. Also, as many mathematicians explain, axiomatization usually comes at the end of the process and not the beginning (Cellucci, 2002).

Now, regarding Harrison's second point, he points to the fact that mathematics is applied in society so issues of precision and reliability in mathematics, as well as computer science and engineering, are important as they can have pragmatic consequences. Hence, it is paramount that not only mathematics should be checked for correctness by computer programs, but also computer programs should be checked for correctness as well. Harrison recognizes the difficulties in this, since computer proof-correctness programs could be usually long and tedious with few people understanding them, yet, as he claims, that should not be considered as an argument against formal verification of a proof. To

\footnotetext{
${ }^{2}$ Harrison mentions D'Alambert's false proof of the Fundamental Theorem of Algebra in 1746. One could add Gauss' incomplete first proof in 1799 of the same theorem.

${ }^{3}$ A nice presentation of that using the quaternions as an example is in Papastavrides (1983), where the author shows the interplay of observation, experimentation, imagination and proof in a famous mathematical discovery.
} 
the contrary, he suggests that we should invest and improve even more our computer methods.

But, the question of reliability still stands, and if the point is to be sure of a proof by mechanically checking it, then how can one be sure of the program that checks the proof? Considering that computer checking is long and tedious, certainly longer and harder than human checking, does not that defeat the purpose of demanding efficiency? For example, the proof of Fermat's Last Theorem is quite long and few mathematicians have read and understood it. Some could have doubts regarding its validity, correctness, etc., and that is quite understandable. But, writing a complex program ten times longer, that also few people understand it, in order to check the theorem, is it something reasonable to pursue? Why not giving incentives, as one could suggest, to say ten mathematicians in humanly verifying the proof?

The first automated theorem prover, known as the "Logic Theory Machine" was developed in the 60's by Newell and Simon (1956). It mimicked the logical skills of a human, but it dealt only with theorem proving from propositional logic ${ }^{4}$. The first computer proof assistant in mathematics was used in the 70's by Appel and Haken in the proof of The Four-Color Theorem (improved in the 90's by Robertson et al.), in which a large number of case checking and calculations was done by the computer. That caused a big controversy on what ultimately a proof is and whether computer proofs could be considered proofs. In 2005, Gonthier $(2005 ; 2008)$ gave a formal proof of the Four-Color Theorem using the proof assistant Coq which automates the whole proof process itself. Also, in the 90's, Hales gave a large computer assisted proof of Kepler's Conjecture which, as we mentioned in previously, he proved in 2006 and formally proved in 2017 using the proof assistant HOL Light. About one hundred other important theorems were formalized $^{5}$, including some in the undergraduate level (e.g. the Fundamental Theorem of Calculus). So, advocates of formal proof would say that this practice is doable and useful, and a natural part of the scientific development and progress.

But, even though there is no doubt that these are important logical and technological achievements, all the above formalized proofs still remain philosophically controversial. First, one must distinguish between proof verification and proof discovering. Proof assistants are formal syntactical systems based on deductive logic that can be used to check whether a set of premises imply a conclusion, independently of content and semantics. Discovery requires more than logical deduction, for example observation, intuition, etc., and not all proofs are deductive. Finally, even though important theorems have been formalized, it does

\footnotetext{
${ }^{4}$ For example, it proved several theorems from Russell's and Whitehead's Principia Mathematica. Another interesting program was Lenat's program AM ("A Mathematician") in the 70's, which exhibited also some creative behavior as it was based on some general heuristics. Nevertheless, AM has its drawbacks too. See more here: https://bit.ly/2Z4fSEa.

${ }^{5}$ Such as, the First Incompleteness Theorem (by Shankar, Boyer-Moore system, 1986), the Fundamental Theorem of Calculus (by Harrison, HOL Light system, 1996), the Fundamental Theorem of Algebra (by Milewski, Mizar system, 2000), the Prime Number Theorem (by Avigad et al., Isabelle system, 2004), the Four Color Theorem (by Gonthier, Coq system, 2005), the Kepler's Conjecture (by Hales, HOL Light system, 2017), etc. (Wiedijk, 2008; see also: https://bit.ly/2MV0 O9D).
} 
not mean that all theorems can be formalized (Harrison, 2008, p. 1403-1404). There are also technical issues which are not in accordance with mathematical practice. For example, HOL Light and Mizar systems define $\frac{1}{0}=0$, even though it is actually undefined, because the systems' functions cannot account for "undefined" and the algorithms require an input in order to run (Wiedijk, 2008).

\section{In Class}

The emphasis given on the foundations of mathematics in the first half of the $20^{\text {th }}$ century, and the rise of programming, automation and computers in the 60's, that we described in the previous section, naturally affected education as well. As Hana says:

"The hallmark of the mathematics curriculum adopted in the sixties was an emphasis on formal proof. Among the manifestations of this emphasis were an axiomatic presentation of elementary algebra and increased classroom attention to the precise formulation of mathematical notions and to the structure of a deductive system." (Hana, 1989, p. 20)

This "new mathematics", as it was usually called, was criticized in the 80 's by Hana (1983, 1989) Kitcher (1984), Davies (1986), Tymoczko (1986) and others, and educators we forced to modify the curriculum de-emphasizing formalities, rigor and proof, and emphasizing more examples and applications. It has been debatable since then, if that was the right approach that should had been followed, as complains were raised later regarding the coherence of the material taught and the impact of reducing rigor and proof had on the critical skills of the students. But, what did some empirical studies show? Deer (1969) found that teaching an explicit unit on logic did not have any effect in improving students' abilities to prove geometric theorems. Cheng at al. (1986) found that college students who took introductory logic had no advantage over students who did not take the course in solving the Wason's Selection Task, yet using concrete examples does improve students' reasoning abilities. On the other hand, Platt (1967) and Mueller (1975) showed that teaching logic was beneficial to geometry students, especially if the logic was covered in context. Also, Durand-Guerrier and Arsac (2009), Durand-Guerrier et al. (2012) and Epp (2003; 2009) claimed that logic is a useful tool in mathematics, yet it should be presented "in a manner that continually links it to language and to both real-world and mathematical subject matter" (Epp, 2003, p. 895). Similarly, as Durand-Guerrier et al. said, "teaching logic as an isolated subject generally appears to be inefficient in developing reasoning abilities" (Durand-Guerrier et al., 2012, p. 375).Hence, it seems to me that a safe conclusion to be drawn from the above is that logic is useful but it should be done in context.

In my experience from teaching discrete mathematics, I certainly see the relevance of logic to mathematics, but I also noticed the following: 
a. Students have conceptual difficulties with the semantics (e.g. " $\wedge ", " \rightarrow "$, etc.) and the scope of propositional logic. For example, some of the connectives seem ambiguous or non-sensical. In particular, students struggle with the conditional $" \rightarrow "$ and its truth values ${ }^{6}$. The fact that propositions $p$ and $q$ could be false yet the proposition $p \rightarrow q$ is true is not something that the students can empirically easily accept, especially when $p$ and $q$ are unrelated. And justifiably so. Neither this logical fact is something that the students use much in proper proofs. Because in mathematical proofs, we are mainly interested in starting from true premises and arriving at true conclusions and in starting from true premises to false conclusions when disproving. The case when the premises are false is usually deemed irrelevant.

b. The formal aspects of logic are quickly dropped, as they are unnecessary. Students have already difficulties with informal proofs, so adding extra formalities and complicating things even further seems anti-pedagogical. Even the few logical rules the students need for mathematical proofs could be summarized and included in the beginning of the proof section, without much harm done. Much of the previous material, especially on propositional logic, could easily be omitted. One could simply start with minimal logical rules and the basic axioms of the subject being studied ${ }^{7}$. See (Suppes, 1965).

Regarding (b), and motivated by some of the research done already, we also asked some of our students' input on the matter in a short survey. In two questionnaires given to 45 students in two discrete mathematics courses, we asked their opinions on some issues related to formal and informal proof and recorded their responses (see Appendix). In particularly, in Questionnaire A, students were given a formal and an informal proof of the same theorem and were asked which they find more rigorous, which they understand better, which is more explanatory, etc. In Questionnaire B, students were given a pictorial proof (without words) and an informal proof of the same theorem and were asked the same questions as above. Their responses are summarized in the Table 1.

Table 1. Responses to the Questionnaires

\begin{tabular}{|l|c|c|c|c|}
\hline Questionnaire A & Question 1 & Question 2 & Question 3 & Question 4 \\
\hline Proof 1 & $88.8 \%$ & $91.1 \%$ & $84.4 \%$ & $22.3 \%$ \\
\hline Proof 2 & $11.2 \%$ & $8.8 \%$ & $15.6 \%$ & $77.7 \%$ \\
\hline & & & & \\
\hline Questionnaire B & Question 1 & Question 2 & Question 3 & Question 4 \\
\hline Proof 1 & $4.5 \%$ & $6.7 \%$ & $22.3 \%$ & $26.7 \%$ \\
\hline Proof 2 & $95.5 \%$ & $93.3 \%$ & $77.7 \%$ & $73.3 \%$ \\
\hline
\end{tabular}

\footnotetext{
${ }^{6}$ More on the "paradoxes" of the conditional see Lewis (1917); Farrell (1979); Mansur (2005). Also, for students' difficulties with the conditional see Hoyles and Küchemann (2002); Romano and Strachota (2016).

${ }^{7}$ An important issue could be raised here, on whether content matters in teaching proofs. According to some educators it does. See Savic (2017).
} 
Surely, one could observe the following:

1. Indeed, the sample was small (45 students) and the conclusions are simply suggestive, not conclusive. Nevertheless, as the survey was more qualitative than quantitative, and many students explained the reasons for their responses, we do get a clear glimpse of their opinions on proof.

2. In Question A.1, contrary to what one would expect, most students found Proof A.1(informal proof)more rigorous than Proof A.2 (formal proof). Considering their comments, an explanation for that could be that the students relate rigor with understanding. Something that they do not really understand clearly, it is perhaps pointless to deem it as rigorous. Similarly, in Question B.1, students stated that Proof B.2 (informal proof) is more rigorous than Proof B.1 (pictorial proof) due to the use of algebra.

3. In Question A.2, overwhelmingly most students found Proof A.1 (informal proof) more explanatory than Proof A.2 (formal proof). As many students explained the first proof is easier to follow and understand and shorter. Similarly, in Question B.2, students stated that Proof B.2 (informal proof) is more explanatory than Proof B.1 (pictorial proof) due to the use of algebra, words, etc. On the contrary, in an older questionnaire (see Questionnaire C, in Appendix), more students found the Euler Diagram more explanatory than the formal proof in understanding the validity of an argument.

4. In Question A.3, most students found Proof A.1 (informal proof) more prompting to explore further similar questions than Proof A.2 (formal proof). Although not many clear reasons were given for that, some students stated that the informal proof was easier and the same reasoning could be used to deal with other similar questions and build similar examples. One student said that algebra related to everything in mathematics, so it was a better tool to explore things further than diagrams. Similarly, in Question B.3, students stated that Proof B.2 (informal proof) was more prompting than Proof B.1 (pictorial proof).

5. In Question A.4, most students found Proof A.2 (formal proof) more appropriate for computers than Proof A.1 (informal proof). As some students said, that is because the formal proof follows order and is written line by line, which is perhaps their way to say that it is more deductive. On the other hand, in Question B.4, students said that Proof B.2 (informal proof) is more appropriate for computers than Proof B.1 (pictorial proof) because computers do not understand images and prefer symbols.

6. Overall, students found the informal proof more rigorous than both the formal and pictorial proofs. More rigorous than the formal proof because it did not contain unnecessary information and formalism, and more rigorous than the pictorial proof because it contained essential information, notation and explanations. It seems that the students followed the middle ground. Also, the students found the informal proof more explanatory than both the formal and pictorial proofs. Apparently, even a minimal use of natural language plays an important role in understanding, as only symbols or figures are not enough. Finally, most students believe that more precision, order and symbolism relates more to computers. 


\section{Logic in Greek and Cypriot High Schools}

What is the status of logic in Greek and Cypriot high schools? In Greece, at the end of the $19^{\text {th }}$ century logic was taught in high schools but it had a more theoretical than analytical nature. It emphasized syllogistic logic and lacked symbolism. Even though it mentioned proofs (deductive and inductive proofs) the examples did not usually come from mathematics, with some exceptions from geometry. Also, logic was not part of introductory sections in mathematics books.

Figure 2. A Logic Book for Greek High Schools from 1906

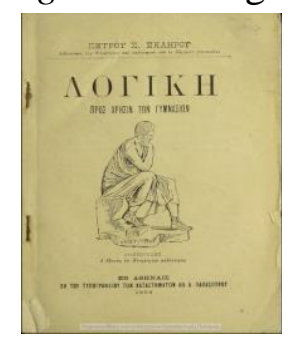

Source: Skliros, 1906.

In the 70-80's logic, as we cover it today, was incorporated in some mathematics books usually as an introductory chapter. It covered the basics on propositional and predicate logic, and the examples were mathematical. Proofs were covered and, interestingly, they were something between the formal and informal proof that we described (see Figure 3).

Figure 3. The Proof of the Same Example we Mentioned Previously MAPAAEITMA

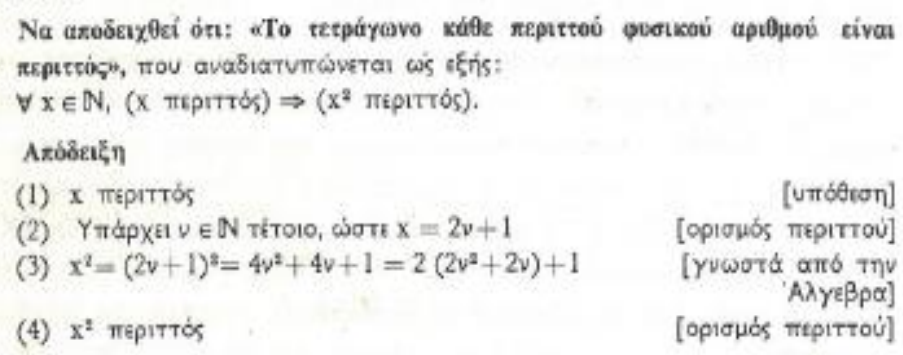

[utrótion] [орібй́́s періттоن'] $[\gamma$ [ [opiquós mepitroú]

Source: Varouchakis et al., 1983, p.26.

A proper logic text is a book by Kyriakopoulos (1977), in which propositional and predicate logic is covered inall detail and all important connections to mathematics are mentioned. As a matter of fact, in regards to our discussion on formal-informal proof here, Kyriakopoulos interestingly stated ${ }^{8}$ that:

"[...] in mathematics, the proofs of propositions are not presented in the form of formal proofs, that we saw in the previous chapters. Formal proofs are long and therefore, not only cumbersome, but also time consuming. Due to that and because in mathematics logic is considered known, in the proofs of propositions of a

\footnotetext{
${ }^{8} \mathrm{My}$ translation.
} 
mathematical theory, not all steps are mentioned and the logic rules are not particularly emphasized." (Kyriakopoulos, 1977, p. 160-170)

Nowadays, elements of logic are included in an introductory chapter of Andreadakis et al. (1998) in the $4^{\text {th }}$ year in high school, but are usually omitted. Some methods of proof (direct and by contradiction) are discussed in chapter two of the same book. In the $5^{\text {th }}$ year of high school, several proofs in number theory (including induction) are covered in chapter four of Adamopoulos et al. (1998). Finally, in the $6^{\text {th }}$ year of high school, there is an optional logic course (Anapolitanos et al., 1999) which is not mathematically oriented and is never taught.

In Cyprus, a relatively extensive chapter on logic and proofs was introduced in high schools in 2017. That was a significant change as since the 80'slogic was not covered and few proofs were done in high school ${ }^{9}$. The chapter is covered in the $5^{\text {th }}$ year of high school, in chapter one of Demetriou et al. [2017]. As it is stated in the book ${ }^{10}$ :

"The book has a two-fold role to fulfill: to introduce the student in the syllogistic that is expressed by the unsurpassable logico-inductive system of mathematics and also to respond to the modern mathematical demands. All the material included in the present book, $[\ldots]$ is intended on the one hand to help the students understand the mathematical logic and thought and on the other to contribute to the mathematical education of the country." (Demetriou et al., 2017)

I am not sure if the book achieves its objectives and it certainly has its problems, ${ }^{11}$ yet it looks like a step in the right direction for Cyprus' educational state of affairs. It contains informal proofs, it does not distract student with unnecessary logical formalities, and it is kept relevant to mathematics.

It seems that Greek and Greek-Cypriot curriculums are diverging regarding logic, but converging regarding proof. I am not aware of what, if any, studies were considered in order to decrease or increase logic in the two curriculums and what

\footnotetext{
${ }^{9}$ Before the 80's, Cyprus was basically following the Greek educational system and books. Some Greek books are still used in Cyprus even today.

${ }^{10}$ My translation.

${ }^{11}$ For a book with twelve authors much more was expected. It was criticized unofficially on the

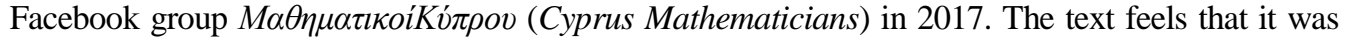
written in a rush and contains some mistakes. For example, negations of quantified propositions are poorly discussed (p.23), there are omissions whether a number is "real" or "integer" in examples (p.11), numerical errors (p.13), etc. There seems also to be a confusion regarding "propositions" and "propositional functions" (p.24). Kyriakopoulos also points out to a misunderstanding of "necessary" and "sufficient" conditions in an example that uses Bernoulli's Inequality (p.51). All the above and some suggestions/corrections are included in an unpublished article by Theodoros Tsaggaris, posted in the group above in 2017. I am not aware if some or any material in the book were improved later.
} 
objectives are expected to be achieved ${ }^{12}$. Nevertheless, it does not seem to me that either curriculum is aware of the conceptual gaps between propositional logic and mathematics. Truth tables and syllogisms are not very useful when we prove things in mathematics. Propositional logic is at best a "warm up" to the predicate logic, which is indeed the appropriate logic for mathematics, and several things from the former could be omitted. From predicate logic some things are important and could perhaps be covered before the section on mathematical proofs. The two curriculums though do keep the formalities to a minimum, since increasing the formalities in proofs, as we argued, does not improve proof doing or understanding and it might even hinder mathematical experience and process.

\section{Conclusion}

The basics of predicate logic form the foundation of mathematics and proof lies at the heart of mathematical practice. Yet, even though mathematical logic is required in mathematics, its strict symbolism and formal rules are rarely used in mathematics whose mathematical symbolism, language and methods are rigorous yet less formal. The difference of these two approaches (formal-informal) affects doing, teaching and learning mathematics. Some argue that a proof is complete only if it is done formally. Others insist that requiring formalities actually inhibits mathematical activity and progress. Formal proof checking and verifying is tediously long and is nowadays done by powerful computer programs with minimal errors, but we are still far from computerized proof making. Some also doubt whether formalizing mathematics serves any mathematical or pedagogical purpose.

In class experience from teaching Discrete Mathematics showed that some of the sections and some of the formalities of logic, taught usually in the beginning of the course, are not necessary for the subsequent important chapters on proof. Also, empirical evidence from students' responses revealed that to a large extend students relate rigor with understanding and not formalism. They related formalism more to computer reasoning and natural language to human reasoning. As students also found informal proofs more rigorous than pictorial proofs, because it contained essential information, notation and explanations, it seems that the students followed a middle ground indicating that even a minimal use of natural language plays an important role in understanding as only symbols or figures are not enough.

\footnotetext{
${ }^{12} \mathrm{As}$ we have seen, studies in general are not in agreement on the relevance of logic in mathematical understanding and performance. The Greek studies on this topic are scarce, and I found only few related articles on proof mainly from the Proceedings of the $4^{\text {th }}$ and $6^{\text {th }}$ Pan-Hellenic Conferences of the Union of Researchers in Mathematics Education (Ev.E.A t.M). The most relevant article I found was an unpublished and undated article entitled "The Role of Logic in Teaching, Learning and Analyzing Proof" by Morou A. and Kalospyros N., which most likely was written after 2008. The authors examine "whether students in upper secondary schools can improve their reasoning and proof abilities by taking an introductory course in logic" (Morou and Kalospyros), and they conclude that they could. I contacted the authors for more details, but I never received a response.
} 
Finally, what is the situation regarding logic in Greek and Greek-Cypriot high schools? We traced historically the curriculum changes regarding logic from the 1900 's and we saw that today Greek and Greek-Cypriot curriculums are diverging on the teaching of logic, but converging regarding proof. Neither curriculum seems to be aware of the conceptual gaps between logic and mathematics, and it is unclear whether they achieve their objectives. Nevertheless, both include logic as a chapter in a mathematics course, keeping it relevant to mathematics and keeping formalities to a minimum.

\section{Acknowledgments}

The author would like to thank Andrei Zavaliy and George Chailos for their valuable comments on the paper, Mohamad Abdallah for his help with the class data collection, Theodoros Tsangaris for his comments on the book Mathematics B' Lyceum, and Babis Stergiou and Christos Lefkonitziatis for communicating to me some of the curriculum historicals related to the paper.

\section{References}

Adamopoulos, L., Viskadourakis, V., Gavalas, D., Polizos, G., \& Sverkos, A. (1998). Mathematics B' Lyceum. Athens: ITYE.

Anapolitanos, D., Gavalas, D., Demis, A., Dimitrakopoulos, K., \& Karasmanis, V. (1999). Logic: Theory and Practice. Athens: ITYE.

Andreadakis, S., Katsargiris, B., Papastavridis, S., Polizos, G., \& Sverkos, A. (1998). Algebra and Elements of Probability. Athens: ITYE.

Aristotle (2012). The Organon. In The Works of Aristotle on Logic, R.B. Jones (ed.). Translations by E. M. Edghill, A. J. Jenkinson, G. R. G. Mure, W. A. PickardCambridge. Create Space Independent Publishing Platform.

Bellucci, F., \& Pietarinen, A.V. (2018). Internet Encyclopedia of Philosophy. Retrieved from https://bit.ly/2PaYuy7.

Cellucci, C. (2002). Introduction to Filosofia e Matematica. In 18 Unconventional Essays on the Nature of Mathematics, R. Hersh (ed.), 17-36. NY: Springer.

Cheng, P.W., Holyoak, K.J., Nisbett, R.E., \& Oliver, L.M. (1986). Pragmatic vs. Syntactic Approaches to Training Deductive Reasoning. Cognitive Psychology, 18, 293-328.

Davies, P. (1986). The Nature of Proof. Proceedings of the $5^{\text {th }}$ International Congress on Mathematical Education, edited by M. Carss. NY: Springer.

Deer, G.W. (1969). The Effects of Teaching an Explicit Unit in Logic on Students' Ability to Prove Theorems in Geometry. Doctoral thesis, Florida State University.

Demetriou, T., Ioannou, I., Karandanos, D., Konstantinidis K., Loizias S., Matheou, K., Papagiannis K., Paragiou, Th., Sergidis, M., Stilianou, A., Timotheou, S., \& Hatzigeorgiou, E. (2017). Mathematics B' Lyceum, vol. A. Nicosia: Pedagogical Institute of Cyprus.

Durand-Guerrier, V., \& Arsac, G. (2009). Analysis of Mathematical Proofs: Some Questions and First Answers. In Proof and Proving in Mathematics Education (ICMI Study 19), G. Hanna, M. De Villiers, F.L Lin., \& F.J. Hsieh (ed.), 148-153. The Department of Mathematics, National Taiwan Normal University Taipei, Taiwan. 
Durand-Guerrier, V., Boero, P., Douek, N., Epp, S., \& Tanguay, D. (2012). Examining the Role of Logic in Teaching Proof. In Proof and Proving in Mathematics Education (ICMI Study 19), G. Hanna, M. De Villiers (ed.), 369-389. NY: Springer.

Epp, S. (2003). The Role of Logic in Teaching Proof. The American Mathematical Monthly, 110(10), 886-899.

Epp, S. (2009). Proof Issues with Existential Quantification. In Proof and Proving in Mathematics Education (ICMI Study 19), G. Hanna, M. De Villiers, F.L. Lin, \& F.J. Hsieh (eds.), 154-159. The Department of Mathematics, National Taiwan Normal University Taipei, Taiwan.

Farrell, R.J. (1979). Material Implication, Confirmation, and Counterfactuals. Notre Dame Journal of Formal Logic, 20(2), 383-394.

Ferreiros, J. (2001). The Road to Modern Logic. The Bulletin of Symbolic Logic, 7(4), 441-484.

Gonthier, G. (2005). A computer-checked proof of the Four-Colour Theorem. Retrieved from https://bit.ly/2Tw0OxT.

Gonthier, G. (2008). Formal Proof - The Four-Color Theorem. Notices of the American Mathematical Society, 55(11), 1382-1393.

Hadamard, J. (1954).The Psychology of Invention in the Mathematical Field. Dover Publications.

Hales, T.C., \& Ferguson, S.P. (2006). The Kepler Conjecture. Discrete and Computational Geometry, 36(1), 1-269.

Hales, T., Adams, M., Bauer, G., Dang, D.T., Harrison, J., Hoang, T.L., Kaliszyk, C., Magron, V., McLaughlin, S., Nguyen, T.T., Nguyen, T.Q., Nipkow, T., Obua, S., Pleso, J., Rute, J., Solovyev, A., Ta, A.H.T., Tran, T.N., Trieu, D.T., Urban, J., Vu, K.K., \& Zumkeller, R. (2017). A Formal Proof of the Kepler Conjecture. Forum of Mathematics, Pi, 5(e2), 1-29.

Hana, G. (1983). Rigorous Proof in Mathematics Education. Toronto: OISE Press.

Hana, G. (1989). More than Formal Proof. For the Learning of Mathematics, 9(1), 20-23.

Harrison, J. (2008). Formal Proof - Theory and Practice. Notices of the AMS, 55(11), 1395-1406.

Hein, J. (2010). Discrete Structures, Logic, and Computability. Boston: Jones and Bartlett.

Hersh, R. (1997). What is Mathematics Really? NY: Oxford University Press.

Hoyles, C., \& Küchemann, D. (2002). Students' Understandings of Logical Implication. Educational Studies in Mathematics, 51(3), 193-223.

Kitcher, P. (1984). The Nature of Mathematical Knowledge. NY: Oxford University Press.

Kyriakopoulos, A. (1977). Mathematical Logic: With Proofs in Mathematics. Athens: Papadimitropoulou Publishing.

Lakatos, I. (1976). Proofs and Refutations. Cambridge University Press.

Lewis, C. I. (1917). The Issues Concerning Material Implication, The Journal of Philosophy, Psychology and Scientific Methods, 14(13), 350-356.

Mansur, M.N. (2005). The paradoxes of Material Implication, Masters Thesis, Memorial University of Newfoundland.

Marfori, M. (2010). Informal Proofs and Mathematical Rigour, Studia Logica, 96, 261272.

Mueller, D.J. (1975). Logic and the Ability to Prove Theorems in Geometry. Doctoral Thesis, Florida State University.

Morou, A., \& Kalospyros, N. (n.d.). The Role of Logic in Teaching, Learning and Analyzing Proof. Retrieved from https://bit.ly/2Tuz9gQ.

Newell, A., \& Simon, H. (1956). The Logic Theory Machine: A Complex Information Processing System. IRE Transactions on Information Theory, 2(3), 61-79. 
Papastavridis, S. (1983). The Process of Mathematical Discovery [in Greek]. Euklides $\Gamma$, 1, 74-93. Hellenic Mathematical Society.

Platt, L.J. (1967). The Effect of the Use of Mathematical Logic in High School Geometry: An Experimental Study. Doctoral Thesis, Colorado State University.

Polya, G. (1954). Mathematics and Plausible Reasoning, vol. I, II. Princeton University Press.

Polya, G. (1973). How to Solve It. Princeton University Press.

Romano, D.A., \& Strachota, S. (2016). College Students' Understanding of Logical Implication, Open Mathematical Education Notes, 6, 57-64.

Rota, G.C. (1997). The Phenomenology of Mathematical Proof. Synthese, 111, 183-196.

Savic, M. (2017). Does Content Matter in an Introdution-to-Proof Course? Journal of Humanistic Mathematics, 7(2), 149-160.

Skliros, P. (1906). Logic for High School Use. Athens: Papaspirou Publications.

Suppes, P. (1965). The Axiomatic Method in High-School Mathematics. Technical Report No.95, Psychology Series, Institute for Mathematical Studies in the Social Sciences, Stanford University.

Tymoczko, T. (1986). Making Room for Mathematicians in the Philosophy of Mathematics. The Mathematical Intelligencer, 8(3), 44-50.

Varouchakis, N., Adamopoulos, L., Alexandris, N., Papakostantinou, D., Papamikroulis, A. (1983). Mathematics A' Lyceum: Algebra. Athens: OE $\Delta \mathrm{B}$.

Wenzel, M., \& Paulson L. (2006). Isabelle/Iar. In The Seventeen Provers of the World, F. Wiedijk (ed.). Berlin: Springer.

Wiedijk, F. (2008). Formal Proof - Getting Started. Notices of the AMS, 55(11), 14081414. 


\section{Appendix}

\section{Questionnaire A}

Consider the theorem:

\section{The sum of any two odd integers is even.}

Look at the two proofs below and answer the following questions:

\section{Proof1:}

Let $x$ and $y$ be arbitrary odd integers.

Then, there exist integers $m$ and $n$ such that $x=2 m+1$ and $y=2 n+1$.

Adding $x$ and $y$ we get $x+y=(2 m+1)+(2 n+1)=2 m+2 n+2=2(m+n+1)=2 k$, where $k=m+n+1$.

Therefore, $x+y=2 k$, which means that $x+y$ is even.

\section{Proof 2:}

1. $\operatorname{odd}(x)$

premise

2. $\operatorname{odd}(y)$

premise

3. $\exists z(x=2 z+1)$

1 , definition of odd

4. $\exists z(y=2 z+1)$

2, definition of odd

5. $x=2 m+13$, existential instantiation

6. $y=2 n+1$

4, existential instantiation

7. $x+y=2(m+n+1) 5,6$, algebra

8. $\exists z(x+y=2 z) 7$, existential generalization

9. even $(x+y) 8$, definition of even

10. $\operatorname{odd}(x) \wedge \operatorname{odd}(y) \rightarrow \operatorname{even}(x+y) \quad 1-9$, conditional proof

11. $\forall x[\operatorname{odd}(x) \wedge \operatorname{odd}(y) \rightarrow \operatorname{even}(x+y)] \quad 10$, universal generalization

12. $\forall y[\operatorname{odd}(x) \wedge \operatorname{odd}(y) \rightarrow \operatorname{even}(x+y)] \quad 11$, universal generalization

1. Which proof from the ones above establishes the result more rigorously? Why?

2. Which proof from the ones above you understand better and you would communicate to others? Why?

3. Which proof from the ones above prompts you to explore similar questions? Why?

4. Which proof from the ones above is more appropriate for computers? Why? 


\section{Questionnaire B}

Consider the theorem:

$$
(a+b)^{2}-(a-b)^{2}=4 a b, a, b \text { reals. }
$$

Look at the two proofs below and answer the following questions:

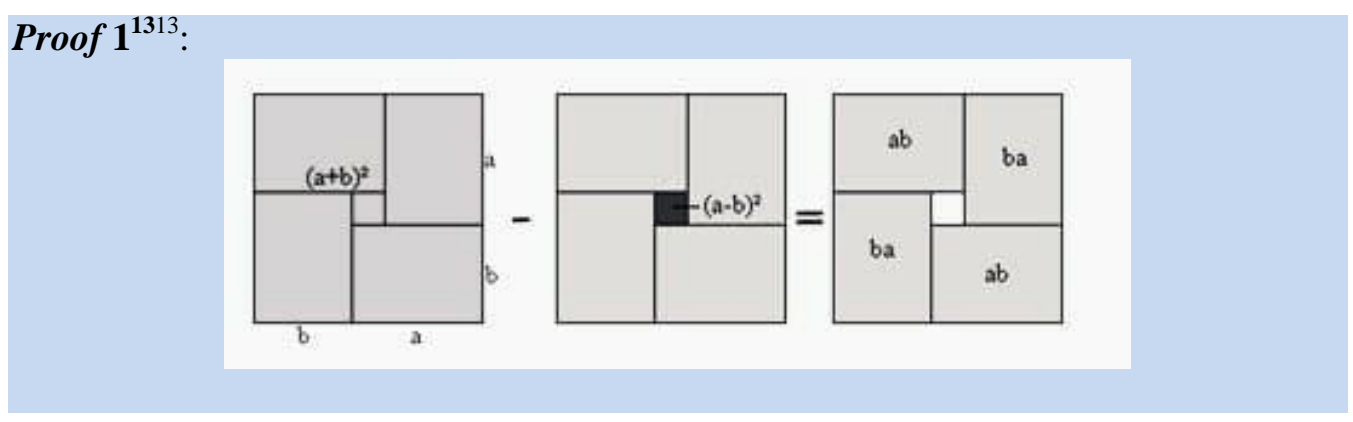

\section{Proof2:}

Using the perfect square identity twice, the left-hand side is:

$$
\begin{aligned}
(a+b)^{2}-(a-b)^{2} & =a^{2}+2 a b+b^{2}-\left(a^{2}-2 a b+b^{2}\right) \\
& =a^{2}+2 a b+b^{2}-a^{2}+2 a b-b^{2} \\
& =4 a b
\end{aligned}
$$

which equals the right-hand side.

1. Which proof from the ones above establishes the result more rigorously? Why?

2. Which proof from the ones above you understand better and you would communicate to others? Why?

3. Which proof from the ones above prompts you to explore similar questions? Why?

4. Which proof from the ones above is more appropriate for computers? Why?

\footnotetext{
${ }^{13}$ Picture taken from https://bit.ly/2Z4N6Dx.
} 


\section{Questionnaire C}

Consider the following valid argument:

All computer science majors are people Some computer science majors are logical thinkers

Therefore, some people are logical thinkers

Which of the two reasonings below helps you more to understand the argument's validity? Why?

\section{Reasoning 1:}

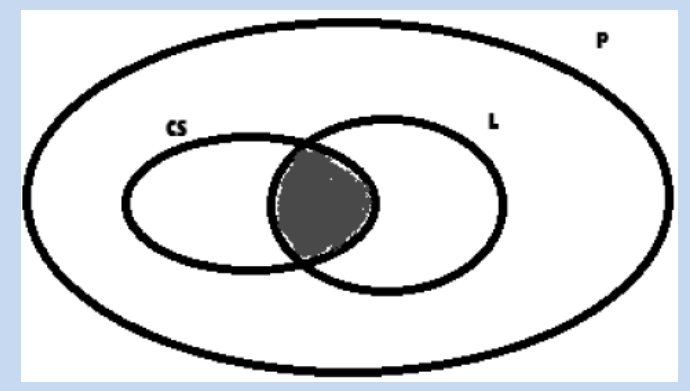

\section{Reasoning2:}

1. $\forall x[C(x) \rightarrow P(x)] \quad$ premise

2. $\exists x[C(x) \wedge L(x)] \quad$ premise

3. $C(c) \wedge L(c) \quad 2$, existential instantiation

4. $\quad C(c) \rightarrow P(c) 1$, universal instantiation

5. $C(c)$

6. $P(c)$

3 , simplification

4,5 , modus ponens

7. $L(c)$

3, simplification

8. $\quad P(c) \wedge L(c) 6,7$, conjunction

9. $\exists x[P(x) \wedge L(x)]$

8 , existential generalization 


\section{Some Students' Responses}

\section{Questionnaire A}

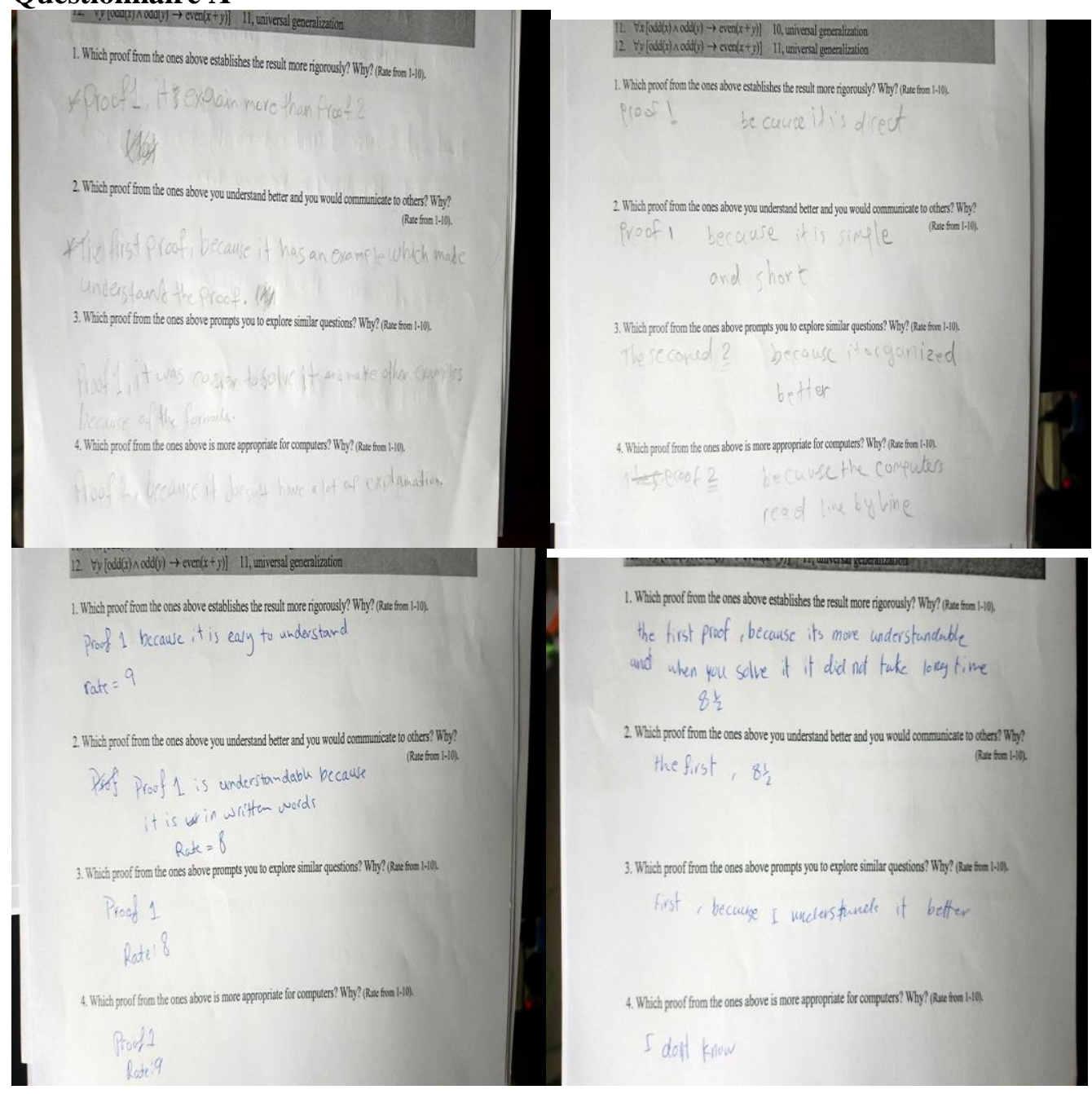

\section{Questionnaire B}
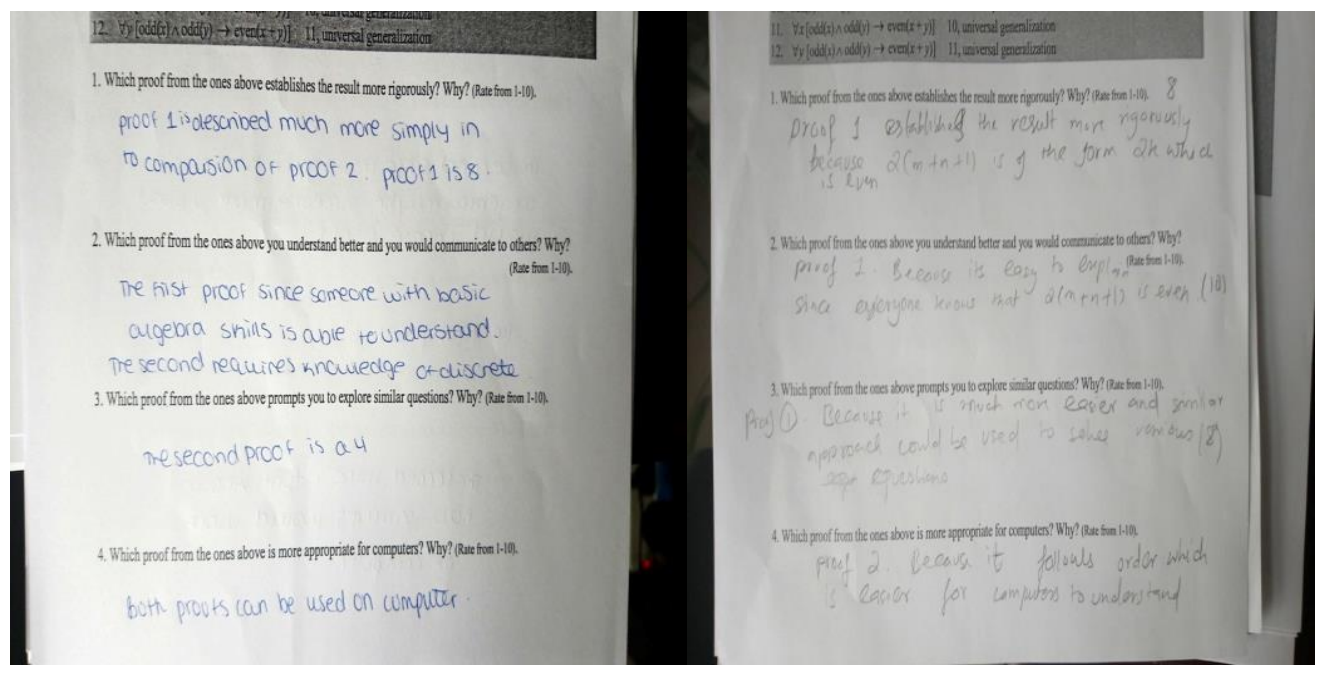


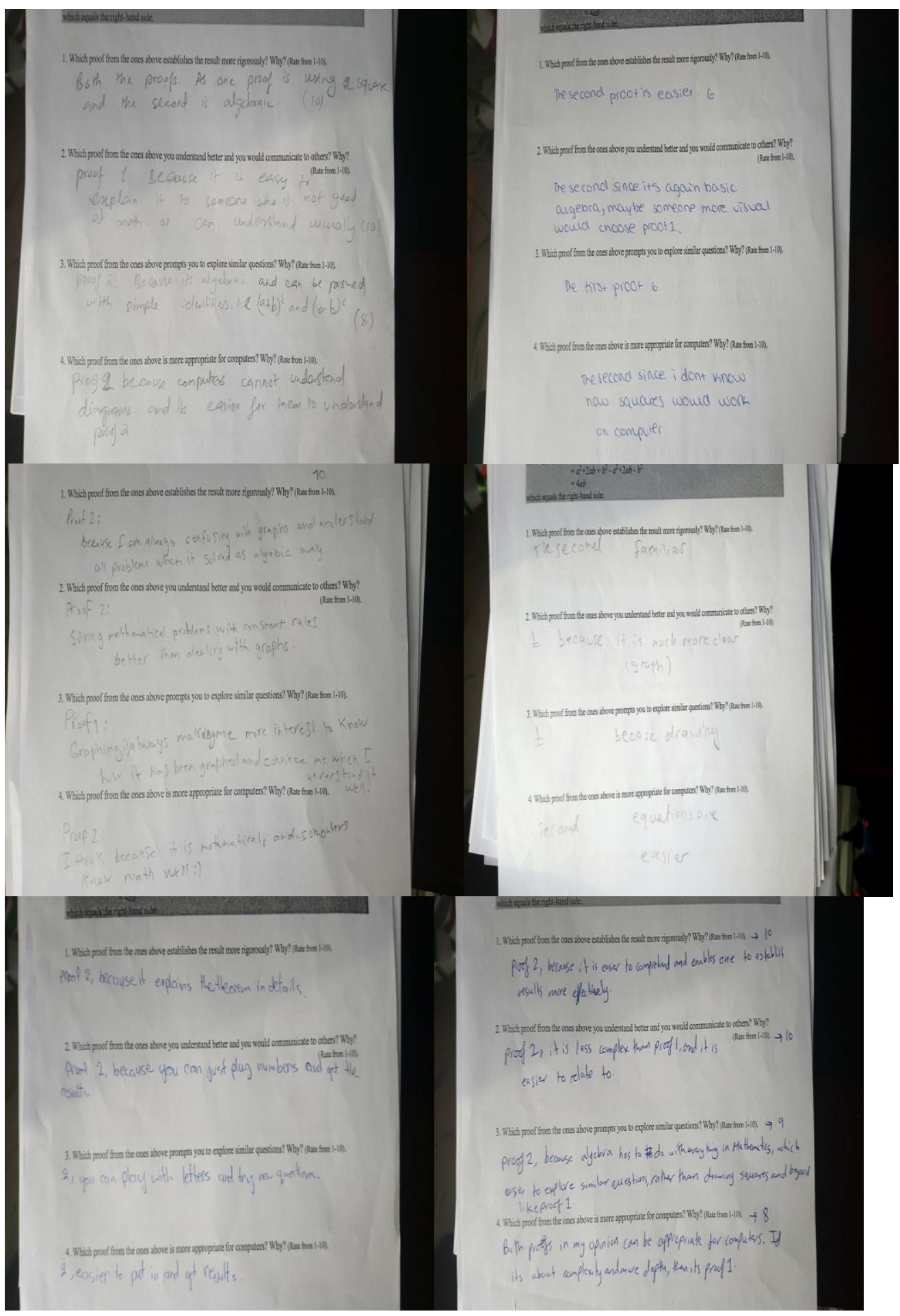




\section{Questionnaire C}

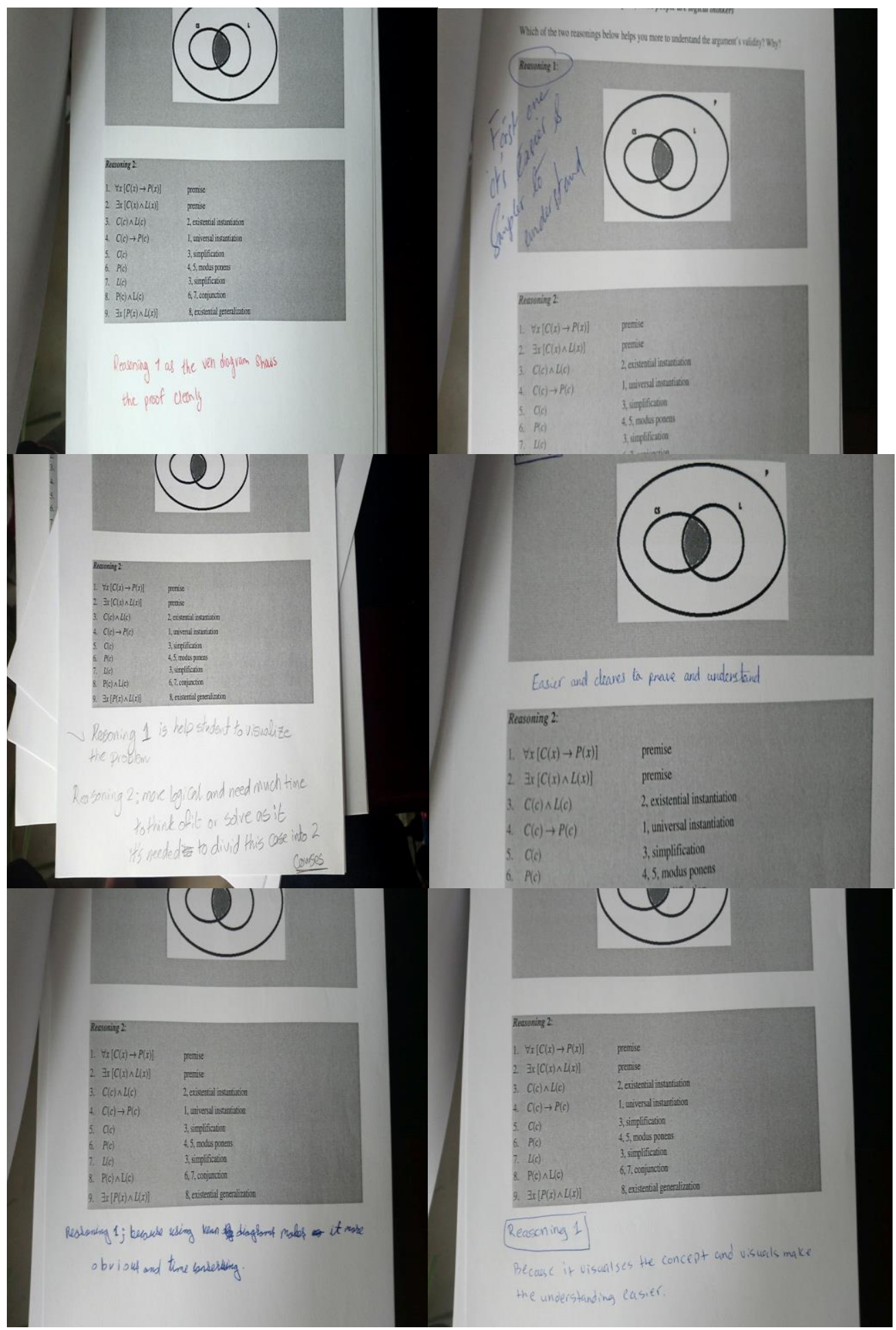


